\title{
A MODEL SELECTION PROCEDURE IN MIXTURE-PROCESS EXPERIMENTS FOR INDUSTRIAL PROCESS OPTIMIZATION
}

\author{
Márcio Nascimento de Souza Leão ${ }^{1}$, Antonio Fernando de Castro Vieira ${ }^{2}$ \\ and Luiz Henrique Abreu Dal Bello ${ }^{3 *}$
}

Received September 19, 2012 / Accepted September 27, 2014

\begin{abstract}
We present a model selection procedure for use in Mixture and Mixture-Process Experiments. Certain combinations of restrictions on the proportions of the mixture components can result in a very constrained experimental region. This results in collinearity among the covariates of the model, which can make it difficult to fit the model using the traditional method based on the significance of the coefficients. For this reason, a model selection methodology based on information criteria will be proposed for process optimization. Two examples are presented to illustrate this model selection procedure.
\end{abstract}

Keywords: mixture experiments, process optimization, information criterion, multicollinearity.

\section{INTRODUCTION}

Formulations obtained from Mixture Experiments (ME) are commonly found in the chemical, pharmaceutical, and food industries, as well as in other industrial segments. In those experiments, the decision variables are the proportions of the components in a mixture and the response is a variable that characterizes the quality of the product, assumed as a function of component proportion. In these experiments, the sum of component proportions is always equal to one. In certain industrial processes, there may be other variables, in addition to the mixture components, that affect the characteristics of the process and must be included in the experiment as factorial designs. Such experiments are called Mixture-Process Experiments (MPEs). Therefore, we intend to determine not only the optimal proportions of the mixture components but also the optimal levels of the process variables.

\footnotetext{
*Corresponding author.

${ }^{1}$ Department of Industrial Engineering, Pontifical Catholic University of Rio de Janeiro (PUC-Rio), Rio de Janeiro, RJ, Brazil. E-mail: mnsleao@yahoo.com.br

${ }^{2}$ Department of Industrial Engineering, Pontifical Catholic University of Rio de Janeiro (PUC-Rio), Rio de Janeiro, RJ, Brazil. E-mail: afcvxx@gmail.com

${ }^{3}$ Brazilian Army Technological Center (CTEx), Rio de Janeiro, RJ, Brazil. E-mail: dalbello@ctex.eb.br
} 
In MEs, it might be necessary to limit the proportion of one or more components that, for technical or practical reasons, cannot be present in all possible proportions. Those limitations of the components, which are very common in industrial cases, may be upper, lower, or a combination of both. Certain combinations of limitations on the proportions of the components may result in a very limited experimental region, which results in collinearity among the covariates of the model, making it difficult to fit the model using the traditional method based on the significance of the coefficients. Consequently, a model selection methodology based on information criteria will be proposed. In order to illustrate this methodology, two examples are used. Matlab ${ }^{\circledR}$ routines were then written for the model selection and the process optimization.

Cornell (2002) is the main reference on ME, being the Chapter 7 dedicated to MPE cases. In it, a comprehensive and detailed exposition can be found. Myers \& Montgomery (2002) dedicate Chapters 12 and 13 to ME and MPE, thus comprising a good introduction to the topic. Piepel (2004) summarizes a survey related to mixture experiments for a period of 50 years, ranging from 1955 to 2004. Prescott et al. (2002) propose a quadratic model as an alternative to the models traditionally used in ME (Scheffe models). Cornell (2000), Cornell (2002, Chapter 6), Cornell \& Gorman (2003) and Khuri (2005) carried out comparative studies between models that they named as slack-variable models and Scheff'e models. Piepel (2007) compares the CSLM (Component Slope Linear Model) with the SLM (Scheffé Linear Model) and the CLM (Cox Linear Model). They conclude that the models SLM, CLM and CSLM are mathematically equivalent and provide the same statistics for a given ME. The differences lie in the interpretations of their coefficients. Dal Bello \& Vieira (2011b) present a tutorial on mixture-process experiments.

Goos \& Donev (2006) describe an algorithm to plan experiments in blocks involving mixtures. They show that, for restricted and unrestricted experimental regions, the resulting design of experiments is statistically more efficient than the options of experiments in blocks presented in the literature. Goos \& Donev (2007) describe an algorithm to plan split-plot experiments in cases involving mixture and process variables. They use an optimization criterion for the choice of experimental points and show that it is preferable to spread the replications all over the experiment region, instead of concentrating them in central points.

Kowalski et al. (2002), Prescott (2004) and Sahni et al. (2009) analyzed the MPE modeling. Goldfarb et al. (2004a) propose the use of a plot method (variance dispersion plot) for MPE planning. The variance dispersion plot presents a visual way of assessing the variance properties of an MPE within the joint mixture and process area. That information may be used to select experiments with an acceptable variance profile.

Goldfarb et al. (2003), Goldfarb et al. (2004b) and Chung et al. (2007) consider the case where, in addition to the mixture components and process variables (controlled factors), there are uncontrolled factors in the productive process (noise variables), although they may be controlled in laboratory experiments. The authors address models that allow them to choose the controllable variable values (mixture and process) that make the process more robust in relation to the noise variables. 
Dal Bello (2010) Dal Bello \& Vieira (2011a) present a methodology close to the spirit of this article.

A brief introduction to ME and MPE is presented in Sections 2 and 3. In Section 4, the information criteria used in this work are described and the models chosen according to those criteria are presented. In Section 5, we present a model selection methodology with two examples, and we apply this methodology in two other examples. The conclusions are in Section 6.

\section{MIXTURE EXPERIMENTS}

Consider $x_{i}$, the variables that represent the proportions of the $q$ mixture components. Then:

$$
\sum_{i=1}^{q} x_{i}=1 ; \quad x_{i} \geq 0 ; \quad i=1, \ldots, q
$$

In many MEs there are limitations on the component proportions, making the experimental space a sub-region of the original space. Therefore, upper and/or lower limits on the proportions are established, and are represented as follows:

$$
0 \leq L_{i} \leq x_{i} \leq U_{i} \leq 1 ; \quad i=1, \ldots, q
$$

where $L_{i}$ is the lower limit and $U_{i}$ is the upper limit of the component proportion $i$.

When the upper and lower limits on the proportions of one mixture are established, the experimental region is reduced to a sub-region of the original region. In these cases, the coordinates of the sub-regions may be redefined in terms of "pseudo"-components.

The models which are traditionally used in MEs are Scheffé's canonical polynomials (Scheffé, 1958). Scheffé's cubic model is as follows:

$$
\begin{aligned}
C(\boldsymbol{\beta}, \boldsymbol{x})= & \sum_{i=1}^{q} \beta_{i} x_{i}+\sum \sum_{i<j}^{q} \beta_{i j} x_{i} x_{j}+\sum \sum \sum_{i<j<k}^{q} \beta_{i j k} x_{i} x_{j} x_{k} \\
& +\sum \sum_{i<j}^{q} \beta_{i-j} x_{i} x_{j}\left(x_{i}-x_{j}\right)
\end{aligned}
$$

where the $\beta$ s are the model's parameter coefficients. Note that this model does not have the intercept, as it is eliminated by a simplification originating from the basic limitation presented in Eq. (1).

\section{MIXTURE-PROCESS EXPERIMENTS}

An adequate model for $\mathrm{r}$ process variables $z_{1}, z_{2}, \ldots, z_{r}$ involving second-order terms is:

$$
Q(\boldsymbol{\delta}, \boldsymbol{z})=\delta_{0}+\sum_{l=1}^{r} \delta_{l} z_{l}+\sum_{l=1}^{r} \delta_{l l} z_{l}^{2}+\sum \sum_{l<m}^{r} \delta_{l m} z_{l} z_{m}
$$


where the $\delta$ s are the model's parameter coefficients for process variables. The experiment for the process variables may be a factorial design with two or more levels. In order to include terms with the variable $z_{j}^{2}$ in the model, an experiment with at least three levels of each process variable and a total number of points sufficient to fit and test the model is required. In order to fit a model without the variable $z_{j}^{2}$, considering only the main effects of the process variables and the interactions among them, only two levels of each variable are necessary.

We use the form of the simultaneous additive and multiplicative combined model, which includes Scheffé's cubic model for the mixture and the reduced quadratic model, considering only the main effects of the process variables and the interactions among them:

$$
\begin{aligned}
& C(\boldsymbol{\gamma}, \boldsymbol{\delta}, \boldsymbol{x}, \boldsymbol{z})=\sum_{i=1}^{q} y_{i}^{l} x_{i}+\sum \sum_{i<j}^{q} \gamma_{i j}^{l} x_{i} x_{j}+\sum \sum_{i<j<k} \sum_{i j k}^{q} \gamma_{i}^{l} x_{i} x_{j} x_{k} \\
& +\sum_{i<j}^{q} \gamma_{i-j}^{l} x_{i} x_{j}\left(x_{i}-x_{j}\right)+\sum_{l=1}^{r} \delta_{l} z_{l}+\sum \sum_{l<m}^{r} \delta_{l m} z_{l} z_{m} \\
& +\sum_{l=1}^{r}\left[\begin{array}{c}
\sum_{i=1}^{q} \gamma_{i}^{l} x_{i}+\sum \sum_{i<j}^{q} \gamma_{i-j}^{l} x_{i} x_{j}\left(x_{i}-x_{j}\right) \\
+x_{i<j<k} x_{j}+\sum \sum_{i j k}^{l} x_{i} x_{j} x_{k}
\end{array}\right] z_{l} \\
& +\sum_{l<1}^{q}\left[\begin{array}{c}
\sum_{i=1}^{q} \gamma_{i}^{l} x_{i}+\sum_{i<j}^{q} \gamma_{i j}^{l} x_{i} x_{j}+\sum \sum_{i<j}^{q} \gamma_{i-j}^{l} x_{i j k} x_{i} x_{j} x_{k}\left(x_{i}-x_{j}\right) \\
+\sum z_{l} z_{m}
\end{array}\right.
\end{aligned}
$$

where the $\gamma$ s are the parameters for the mixture's combined model including process variables and the $\delta \mathrm{s}$ are the parameters for the process variables. The lower indexes of $\gamma$ refer to mixture variables, whereas the upper ones refer to process variables. The lower indexes of $\delta$ refer to process variables.

\section{INFORMATION CRITERIA AND MODEL SELECTION}

An information criterion that has been widely used in model selection is Akaike's criterion $(A I C)$ (Akaike, 1973).

$$
A I C=-2 \sum_{i=1}^{n} \ln L\left(\hat{\mu}_{i}, y_{i}\right)+2 p
$$

where $y_{i}$ is the $\mathrm{i}^{\text {th }}$ value of the response and $\hat{\mu}_{i}$ is the estimate of $y_{i}$ when a model of $p$ parameters is fitted through maximization of the Log-Likelihood Function (LLF). The term added to LLF, called the penalty function, aims at correcting a bias originating from the comparison of models with different numbers of parameters. Among the several candidate models, the one with the lowest $A I C$ value must be chosen. AIC was developed from Kullback-Leibler distance, which 
is a distance between the true model and the candidate model. Burnham \& Anderson (2002) recommend the use of $A I C$ only when $n / p \geq 40$. Considering a case of responses with normal distribution, the $A I C$ expression may be simplified to give the following:

$$
\begin{aligned}
A I C & =n \ln \left(\hat{\sigma}_{p}^{2}\right)+2(p+1) \\
\hat{\phi}_{p}^{2} & =\frac{\sum_{i=1}^{n}\left(y_{i}-\hat{\phi}_{i}\right)^{2}}{n}
\end{aligned}
$$

where $\hat{\sigma}_{p}^{2}$ is the maximum likelihood estimator of the error variance.

Considering responses with normal distribution and small samples $(n / p<40)$, Hurvich \& Tsai (1989) developed the AICc criterion:

$$
A I C_{c}=A I C+\frac{2(p+1)(p+2)}{n-p-2}
$$

Burnham \& Anderson (2002) recommend the calculation of $A I C$ differences between the candidate models and the model with the lowest $A I C_{c}$ value $\left(A I C_{C_{-} \min }\right)$.

$$
\Delta_{i}=A I C_{c_{-} i}-A I C_{C_{\_} \min }
$$

The calculation methodology for $A I C$ differences may also be used for $A I C_{c}$ differences. $\Delta_{i}$ values can be interpreted easily and allow a quick comparison of candidate models. The higher the $\Delta_{i}$, the less likely it is that the fitted model is the best model according to Kullback-Leibler distance. Burnham \& Anderson (2002) affirm that models with $\Delta_{i}>10$ may be omitted in future considerations and models with $\Delta_{i}$ between 0 and 2 may be regarded as non-different. The calculation of $A I C_{c}$ differences is used in the proposed methodology for model selection which will be used in Section 5.

\section{PROPOSED METHODOLOGY}

In the first stage of this methodology we use the full Scheffé's canonical polynomials for a ME and a combined model for a full MPE. Thus, we obtain all the candidate terms for the model under study. Then, we use the $A I C_{c}$ criterion to select the model with the lowest $A I C_{c}$ according to the number of parameters. Afterwards, we calculate the $A I C_{c}$ differences between the candidate models and the model that has the lowest AICc and we select the non-different models.

Analyzing the non-different models, we choose the model, now named the Base Model, which has the lowest mean-squared error (MSE) and prediction error sum of squares (PRESS).

In the second stage of the methodology we obtain a better model, taking into account the Base Model terms and all terms which are equivalent to the terms of the Base Model. Such Equivalent Terms are created considering Eq. (1), which is the basic restriction of MEs. For example, the term $x_{1} x_{2}$ is equivalent to term $x_{1}\left(1-x_{1}-x_{3}\right)$ or term $\left(1-x_{2}-x_{3}\right) x_{2}$. After determination of all 
the candidate terms (terms of the Base Model and Equivalent Terms), we use the $A I C_{c}$ criterion again in order to select the model with the lowest $A I C_{c}$. Afterwards, we calculate the $A I C_{c}$ differences between the candidate models and model that has the lowest $A I C_{c}$ and we select the non-different models.

Analyzing the non-different models, we choose the model with the lowest PRESS and MSE as the Final Model. The proposed methodology is illustrated through two examples.

\subsection{Example 1}

The problem of Example 1 was presented by Myers \& Montgomery (2002). An adhesive is being formulated for use in an aerospace application. The adhesive consists of a resin $x_{1}$ and two crosslinkers, $x_{2}$ and $x_{3}$. The mixture constraints for these variables are $x_{1}+x_{2}+x_{3}=1$; $0.70 \leq x_{i} \leq 0.90 ; 0.05 \leq x_{2} \leq 0.10 ;$ and $0.05 \leq x_{2} \leq 0.20$.

The adhesive is applied to the components and then the entire assembly is cured for $12 \mathrm{~h}$ at controlled temperature and humidity. The temperature $z_{1}$ and relative humidity $z_{2}$ are process variables that can be controlled by the experimenter. The ranges of theses process variables that experimenters think are appropriate are $40^{\circ} \mathrm{F} \leq$ temperature $\leq 100^{\circ} \mathrm{F}$ and $15 \% \leq$ relative humidity $\leq 85 \%$. The response variable of interest is the pulloff force required to separate the components after curing. It should exceed 40 pounds.

The authors use L-pseudocomponents according to the relation $v_{i}=\frac{x_{i}-L_{1}}{1-L} ; i=1,2, \ldots, q$, where $L=\sum_{i=1}^{q} L_{i}$ and Table 1 presents the experiment.

Where

$\hat{y}_{1}$ : Response obtained by Myers \& Montgomery (2002) model.

$\hat{y}_{2}$ : Response obtained by Final Model 1 in this article.

The model selected by Myers \& Montgomery (2002) presented PRESS, MSE and $A I C_{c}$ equal to $903.20,15.45$ and 122.57, respectively, and is shown in Eq. (11).

$$
\begin{aligned}
\hat{y}= & 40.66 v_{1}+71.95 v_{2}+46.16 v_{3}-30.58 v_{1} v_{3}+9.32 v_{1} z_{1} \\
& -15.49 v_{1} z_{2}+29.92 v_{2} z_{1}-20.18 v_{2} z_{2}+7.43 v_{3} z_{1} \\
& -4.41 v_{3} z_{2}+19.39 v_{1} v_{3} z_{1}-2.60 v_{3} z_{1} z_{2}
\end{aligned}
$$

\subsubsection{Model Selection Methodology}

All the candidate terms for the MPE are the terms in Eq. (5). The model selected according to the $A I C_{c}$ criterion was the following:

$$
\begin{aligned}
\hat{y}= & 40.36 v_{1}+67.32 v_{2}+35.09 v_{3}+11.79 z_{1}-16.19 z_{2}+11.25 v_{3} z_{2} \\
& -3.53 v_{1} z_{1} z_{2}-106.74 v_{2} v_{3}\left(v_{2}-v_{3}\right)+172.09 v_{1} v_{2} v_{3} z_{1} \\
& -45.36 v_{1} v_{2} z_{1} z_{2}+138.49 v_{1} v_{2} z_{1} z_{2}\left(v_{1}-v_{2}\right)
\end{aligned}
$$

A Matlab ${ }^{\circledR}$ routine was then written for the calculation and storage of $A I C_{c}$ values and selection of non-different models considered, that is, those that presented $A I C_{c}$ differences $\left(\Delta_{i}\right)$ between 
Table 1 - The experiment of Example 1 with L-pseudocomponents for mixture components and coded for process variables.

\begin{tabular}{|c|c|c|c|c|c|c|c|c|c|}
\hline Std & Run & $v_{1}$ & $v_{2}$ & $v_{3}$ & $z_{1}$ & $z_{2}$ & Force (lb) & $\hat{y}_{1}$ & $\hat{y}_{2}$ \\
\hline 1 & 26 & 1.000 & 0.000 & 0.000 & -1.000 & -1.000 & 44 & 46.830 & 42.410 \\
\hline 2 & 6 & 1.000 & 0.000 & 0.000 & 1.000 & -1.000 & 70 & 65.470 & 71.010 \\
\hline 3 & 21 & 1.000 & 0.000 & 0.000 & -1.000 & 1.000 & 19 & 15.850 & 17.250 \\
\hline 4 & 8 & 1.000 & 0.000 & 0.000 & 1.000 & 1.000 & 33 & 34.490 & 31.490 \\
\hline 5 & 5 & 0.000 & 0.250 & 0.750 & -1.000 & -1.000 & 48 & 45.958 & 48.273 \\
\hline 6 & 2 & 0.000 & 0.250 & 0.750 & 1.000 & -1.000 & 72 & 75.963 & 74.638 \\
\hline 7 & 30 & 0.000 & 0.250 & 0.750 & -1.000 & 1.000 & 32 & 33.153 & 32.358 \\
\hline 8 & 24 & 0.000 & 0.250 & 0.750 & 1.000 & 1.000 & 59 & 55.358 & 58.723 \\
\hline 9 & 17 & 0.250 & 0.000 & 0.750 & -1.000 & -1.000 & 32 & 32.743 & 33.245 \\
\hline 10 & 23 & 0.250 & 0.000 & 0.750 & 1.000 & -1.000 & 58 & 59.719 & 56.460 \\
\hline 11 & 33 & 0.250 & 0.000 & 0.750 & -1.000 & 1.000 & 21 & 22.283 & 19.125 \\
\hline 12 & 9 & 0.250 & 0.000 & 0.750 & 1.000 & 1.000 & 38 & 41.459 & 38.750 \\
\hline 13 & 11 & 0.750 & 0.250 & 0.000 & -1.000 & -1.000 & 51 & 50.675 & 51.434 \\
\hline 14 & 20 & 0.750 & 0.250 & 0.000 & 1.000 & -1.000 & 76 & 79.615 & 74.956 \\
\hline 15 & 19 & 0.750 & 0.250 & 0.000 & -1.000 & 1.000 & 22 & 17.350 & 16.251 \\
\hline 16 & 22 & 0.750 & 0.250 & 0.000 & 1.000 & 1.000 & 49 & 46.290 & 45.459 \\
\hline 17 & 10 & 0.500 & 0.125 & 0.375 & -1.000 & 1.000 & 17 & 15.132 & 16.087 \\
\hline 18 & 16 & 0.125 & 0.125 & 0.750 & 1.000 & -1.000 & 69 & 67.841 & 67.978 \\
\hline 19 & 32 & 0.125 & 0.125 & 0.750 & -1.000 & -1.000 & 40 & 39.350 & 38.329 \\
\hline 20 & 3 & 0.375 & 0.250 & 0.375 & -1.000 & -1.000 & 37 & 41.289 & 36.113 \\
\hline 21 & 7 & 0.500 & 0.125 & 0.375 & 1.000 & 1.000 & 46 & 42.826 & 43.705 \\
\hline 22 & 29 & 0.375 & 0.250 & 0.375 & -1.000 & 1.000 & 21 & 18.224 & 20.338 \\
\hline 23 & 27 & 0.375 & 0.250 & 0.375 & 1.000 & -1.000 & 82 & 76.215 & 80.948 \\
\hline 24 & 34 & 0.375 & 0.250 & 0.375 & 1.000 & 1.000 & 43 & 49.250 & 48.469 \\
\hline 25 & 15 & 0.625 & 0.000 & 0.375 & -1.000 & -1.000 & 32 & 32.760 & 36.563 \\
\hline 26 & 1 & 0.625 & 0.000 & 0.375 & 1.000 & -1.000 & 60 & 61.021 & 62.470 \\
\hline 27 & 28 & 0.750 & 0.063 & 0.187 & -1.000 & 1.000 & 14 & 13.160 & 15.602 \\
\hline 28 & 4 & 0.750 & 0.063 & 0.187 & 1.000 & 1.000 & 38 & 38.155 & 39.956 \\
\hline 29 & 14 & 0.626 & 0.187 & 0.187 & -1.000 & -1.000 & 45 & 42.680 & 43.160 \\
\hline 30 & 13 & 0.375 & 0.250 & 0.375 & -1.000 & 1.000 & 18 & 18.224 & 20.338 \\
\hline 31 & 18 & 0.125 & 0.125 & 0.750 & 1.000 & -1.000 & 70 & 67.841 & 67.978 \\
\hline 32 & 25 & 0.750 & 0.250 & 0.000 & -1.000 & 1.000 & 10 & 17.350 & 16.251 \\
\hline 33 & 12 & 0.375 & 0.250 & 0.375 & 1.000 & 1.000 & 52 & 49.250 & 48.469 \\
\hline 34 & 31 & 0.750 & 0.250 & 0.000 & 1.000 & 1.000 & 42 & 46.290 & 45.459 \\
\hline
\end{tabular}


0 and 2, as presented in Section 4. According to the $A I C_{c}$ criterion, 23 models are considered non-different. The Model that presents the lowest PRESS (536.67) and MSE (10.44) was selected and it's now named as the Base Model.

The Base Model is shown in Eq. (13):

$$
\begin{aligned}
\hat{y}= & 40.36 v_{1}+67.37 v_{2}+35.10 v_{3}+10.68 z_{1}-16.20 z_{2}+9.86 v_{2} z_{1} \\
& +11.25 v_{3} z_{2}-3.55 v_{1} z_{1} z_{2}-106.69 v_{2} v_{3}\left(v_{2}-v_{3}\right) \\
& -141.28 v_{1} v_{2} v_{3} z_{1}-46.60 v_{1} v_{2} z_{1} z_{2}+138.17 v_{1} v_{2} z_{1} z_{2}\left(v_{1}-v_{2}\right)
\end{aligned}
$$

In this step of the methodology we will consider other models using the Base Model. For this, additional terms are generated from the terms of the Base Model. Table 2 presents the terms equivalent to the Base Model terms.

Table 2 - Equivalent Terms.

\begin{tabular}{|l|l|}
\hline Base Model Terms & Equivalent Terms \\
\hline$v_{2} z_{1}$ & $\left(z_{1}-v_{1} z_{1}-v_{3} z_{1}\right)$ \\
\hline$v_{3} z_{2}$ & $\left(z_{2}-v_{1} z_{2}-v_{2} z_{2}\right)$ \\
\hline$v_{1} z_{1} z_{2}$ & $\left(z_{1} z_{2}-v_{2} z_{1} z_{2}-v_{3} z_{1} z_{2}\right)$ \\
\hline$v_{2} v_{3}\left(v_{2}-v_{3}\right)$ & $\begin{array}{l}\left(v_{2}^{2} v_{3}-v_{2} v_{3}^{2}\right) ; \\
\left(v_{2}^{2}-v_{2}^{3}-v_{1} v_{2}^{2}-v_{3}^{2}+v_{3}^{3}+v_{1} v_{3}^{2}\right)\end{array}$ \\
\hline$v_{1} v_{2} v_{3} z_{1}$ & $\left(v_{2} v_{3} z_{1}-v_{2}^{2} v_{3} z_{1}-v_{2} v_{3}^{2} z_{1}\right) ;$ \\
& $\left(v_{1} v_{3} z_{1}-v_{1}^{2} v_{3} z_{1}-v_{1} v_{3}^{2} z_{1}\right) ;$ \\
& $\left(v_{1} v_{2} z_{1}-v_{1}^{2} v_{2} z_{1}-v_{1} v_{2}^{2} z_{1}\right)$
\end{tabular}

Once all the candidate terms (Base Model terms and Equivalent Terms) for the MPE model are known, we may then use the $A I C_{c}$ criterion again. The model selected is shown in Eq. (14). This model presents PRESS and MSE equal to 586.66 and 11.12, respectively.

$$
\begin{aligned}
\hat{y}= & 40.53 v_{1}+66.44 v_{2}+49.15 v_{3}+11.82 z_{1}-16.16 z_{2} \\
& +10.95 v_{3} z_{2}-71.94 v_{1} v_{3}^{2}-3.57 v_{1} z_{1} z_{2}+174.81 v_{1} v_{2} v_{3} z_{1} \\
& -46.32 v_{1} v_{2} z_{1} z_{2}+139.72 v_{1} v_{2} z_{1} z_{2}\left(v_{1}-v_{2}\right)
\end{aligned}
$$

This model presents higher MSE and PRESS than the model of Eq. (13). However, we will analyze models considered non-different to the model of Eq. (14), as described at the start in Section 5.1.1. The Model that presents the lowest PRESS (515.43) and MSE (10.13) was selected and now, it should be Final Model 1. The Final Model 1 is shown in Eq. (15):

$$
\begin{aligned}
\hat{y}= & 40.54 v_{1}+66.48 v_{2}+49.17 v_{3}+10.71 z_{1}-16.17 z_{2}+9.89 v_{2} z_{1} \\
& +10.95 v_{3} z_{2}-71.95 v_{1} v_{3}^{2}-3.59 v_{1} z_{1} z_{2}+143.90 v_{1} v_{2} v_{3} z_{1} \\
& -47.56 v_{1} v_{2} z_{1} z_{2}+139.40 v_{1} v_{2} z_{1} z_{2}\left(v_{1}-v_{2}\right)
\end{aligned}
$$


Examining the model obtained by Myers \& Montgomery (2002) and Final Model 1 obtained in this article, we observe that the application of the methodology led to a decrease of $43.93 \%$ in PRESS (from 903.20 to 515.43) and a decrease of $34.43 \%$ in the MSE (from 15.45 to 10.13) and kept the number of parameters of the Final Model 1 (12 parameters).

Table 3 shows the $t$-Student test for the Final Model 1 .

Table 3 - Final Model 1 Test.

\begin{tabular}{|c|r|r|r|r|}
\hline Label & Estimate & Std. Error & $t$ value & $p$-value \\
\hline$v_{1}$ & 40.54 & 1.35858 & 29.840 & $<0.0001$ \\
$v_{2}$ & 66.48 & 5.21568 & 12.745 & $<0.0001$ \\
$v_{3}$ & 49.17 & 2.72807 & 18.023 & $<0.0001$ \\
$z_{1}$ & 10.71 & 0.927052 & 11.556 & $<0.0001$ \\
$z_{2}$ & -16.17 & 0.895175 & -18.065 & $<0.0001$ \\
$v_{2} z_{1}$ & 9.89 & 5.49969 & 1.798 & 0.0859 \\
$v_{3} z_{2}$ & 10.95 & 1.86759 & 5.861 & $<0.0001$ \\
$v_{1} v_{3}^{2}$ & -71.95 & 17.8628 & -4.028 & 0.0006 \\
$v_{1} z_{1} z_{2}$ & -3.59 & 1.33296 & -2.690 & 0.0134 \\
$v_{1} v_{2} v_{3} z_{1}$ & 143.90 & 45.4498 & 3.167 & 0.0045 \\
$v_{1} v_{2} z_{1} z_{2}$ & -47.56 & 19.2829 & -2.467 & 0.0219 \\
$v_{1} v_{2} z_{1} z_{2}\left(v_{1}-v_{2}\right)$ & 139.40 & 41.6826 & 3.345 & 0.0029 \\
\hline
\end{tabular}

\subsubsection{Response Optimization}

In the Example 1, a response exceeding 40 pounds is desirable. Several formulations may result in a future response prediction greater than 40 pounds. Consequently, a desirable objective is to maximize the expected value for a future response.

The estimation vector for the coefficients is $\hat{\boldsymbol{\beta}}=\left(\boldsymbol{W}^{\prime} \boldsymbol{W}\right)^{-\mathbf{1}} \boldsymbol{W}^{\prime} \boldsymbol{y}$, the variance-covariance matrix is $\operatorname{var}(\hat{\boldsymbol{\beta}})=\sigma^{2}\left(\boldsymbol{W}^{\prime} \boldsymbol{W}\right)^{-1}$, where $W$ is a matrix $(n \times p)$ whose elements are the mixture components proportion $\left(x_{i}\right)$, the levels of the process variables $\left(z_{i}\right)$ and functions of $x_{i}$ and $z_{i}$ (such as interactions), where $p$ is the number of parameters and $n$ the number of observations.

The general combined model with the inclusion of process variables is represented in a matrix form as

$$
y=W \beta+\varepsilon
$$

For $n$ observations, $y$ is the vector $(n \times 1)$ of observations, $\boldsymbol{\beta}$ is the vector $(p \times 1)$ of coefficients and $\varepsilon$ is the vector $(n \times 1)$ of random errors. In the classical linear model, $\varepsilon$ is considered with multivariate normal distribution, i.e. $\varepsilon \sim N\left(\mathbf{0}, \boldsymbol{I} \boldsymbol{\sigma}^{2}\right)$. The estimated mean response at point $\boldsymbol{w}$ $\left(\boldsymbol{w}^{\prime}\right.$ is a matrix line $\left.\boldsymbol{W}\right)$ is and its variance is

$$
E[\hat{y}(\boldsymbol{w})]=\boldsymbol{w}^{\prime} \hat{\boldsymbol{\beta}}
$$


and its variance is

$$
\operatorname{var}[\hat{y}(\boldsymbol{w})]=\boldsymbol{\sigma}^{2} \boldsymbol{w}^{\prime}\left(\boldsymbol{W}^{\prime} \boldsymbol{W}\right)^{-1} \boldsymbol{w}
$$

The problem may then be formulated as follows:

$$
\max E[\hat{y}(\boldsymbol{w})]=\boldsymbol{w}^{\prime} \hat{\boldsymbol{\beta}}
$$

subject to:

$$
\begin{aligned}
& v_{1}+v_{2}+v_{3}=1 \\
& 0 \leq v_{1} \leq 1 \\
& 0 \leq v_{2} \leq 0.25 \\
& 0 \leq v_{3} \leq 0.75 \\
& -1 \leq z_{1} \leq 1 \\
& -1 \leq z_{2} \leq 1
\end{aligned}
$$

Using a search routine in Matlab ${ }^{\circledR}$, the solution for the problem formulated above was found, considering the model obtained by Myers \& Montgomery (2002) and Final Model 1 obtained in this article. Table 4 presents the optimal values for the components proportions, in L-pseudo components $\left(v_{i}\right)$ and in actual values $\left(x_{i}\right)$ and the optimal values of the process variables, in coded variables $\left(z_{i}\right)$ and in actual values $\left({ }^{\circ} \mathrm{F}\right.$ and $\mathrm{RH}$, respectively).

Table 4 - Solution for the maximization problem of example 1.

\begin{tabular}{|c|c|c|c|c|c|}
\hline Model & $\begin{array}{c}v_{1} \\
(x 1)\end{array}$ & $\begin{array}{c}v_{2} \\
(x 2)\end{array}$ & $\begin{array}{c}v_{3} \\
(x 3)\end{array}$ & $\begin{array}{c}z_{1} \\
\left(\mathrm{~T}\left({ }^{\circ} \mathrm{F}\right)\right)\end{array}$ & $\begin{array}{c}z_{2} \\
(\mathrm{RH}(\%))\end{array}$ \\
\hline Myers and & 0.7400 & 0.2600 & 0.000 & 1.0 & -1.0 \\
Montgomery & $(0.8480)$ & $(0.1020)$ & $(0.0500)$ & $(100.0)$ & $(15.0)$ \\
\hline Final Model 1 & 0.4638 & 0.2600 & 0.2762 & 1.0 & -1.0 \\
& $(0.7928)$ & $(0.1020)$ & $(0.1052)$ & $(100.0)$ & $(15.0)$ \\
\hline
\end{tabular}

Table 5 compares the PRESS, MSE, $A I C_{c}$, the response prediction and the variance of a new response for both models. Analyzing the Table 4, we observe that the model obtained in this article presents lower value for PRESS, MSE and $A I C_{c}$, emphasizing that was obtained a higher response prediction with a lower variance of a new response.

Table 5 - Comparison of two models.

\begin{tabular}{|c|c|c|c|c|c|}
\hline Model & PRESS & MSE & $A I C_{c}$ & $\operatorname{var}[\hat{y}(\boldsymbol{w})]$ & $E[\hat{y}(\boldsymbol{w})]$ \\
\hline Myers \& Montgomery & 903.20 & 15.45 & 122.57 & 21.5172 & 80.1773 \\
\hline Final Model 1 & 515.43 & 10.13 & 108.13 & 16.0093 & 82.3177 \\
\hline
\end{tabular}




\subsubsection{Model Adequacy}

The use of studentized residuals to check the normality is recommended by Myers \& Montgomery (2002). The studentized residuals $\left(r_{i}\right)$ are defined as follows:

$$
r_{i}=\frac{e_{i}}{\sqrt{\hat{\sigma}^{2}\left(1-h_{i i}\right)}}
$$

where $e_{i}=y_{i}-\hat{y}_{i}$ and $h_{i i}$ are elements of the hat matrix diagonals $\boldsymbol{H}=\boldsymbol{W}\left(\boldsymbol{W}^{\prime} \boldsymbol{W}\right)^{-1} \boldsymbol{W}^{\prime}$.

Figure 1 and 2 present the diagnosis plots used to check the adequacy of the Final Model 1 (a) and the model obtained by Myers \& Montgomery (b).

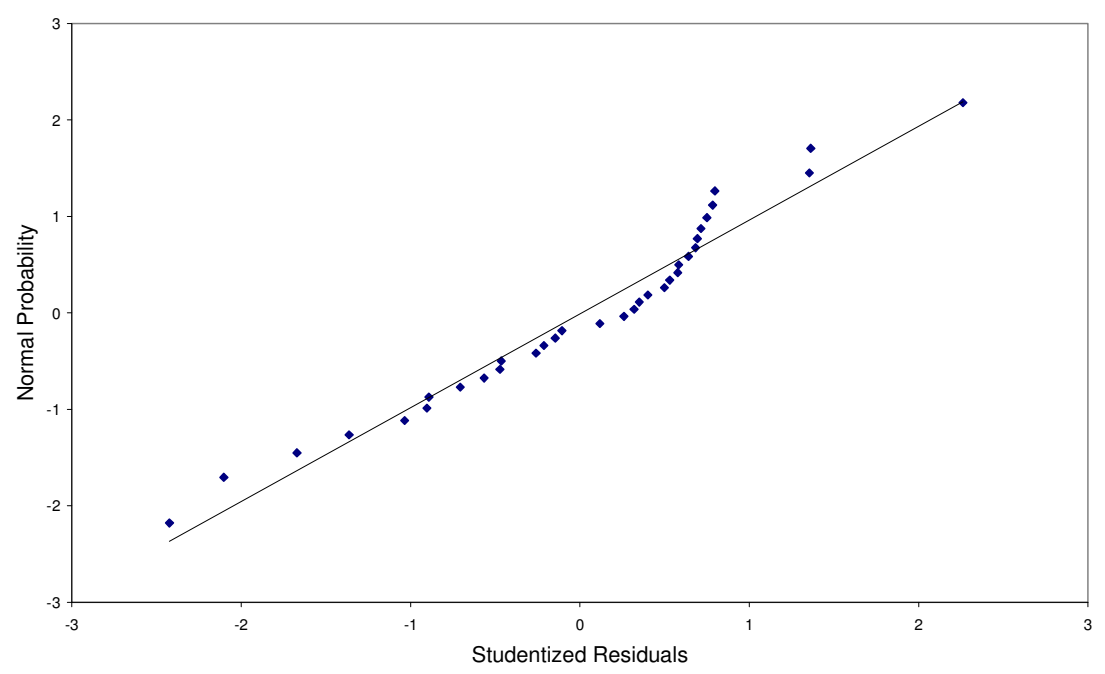

(a)

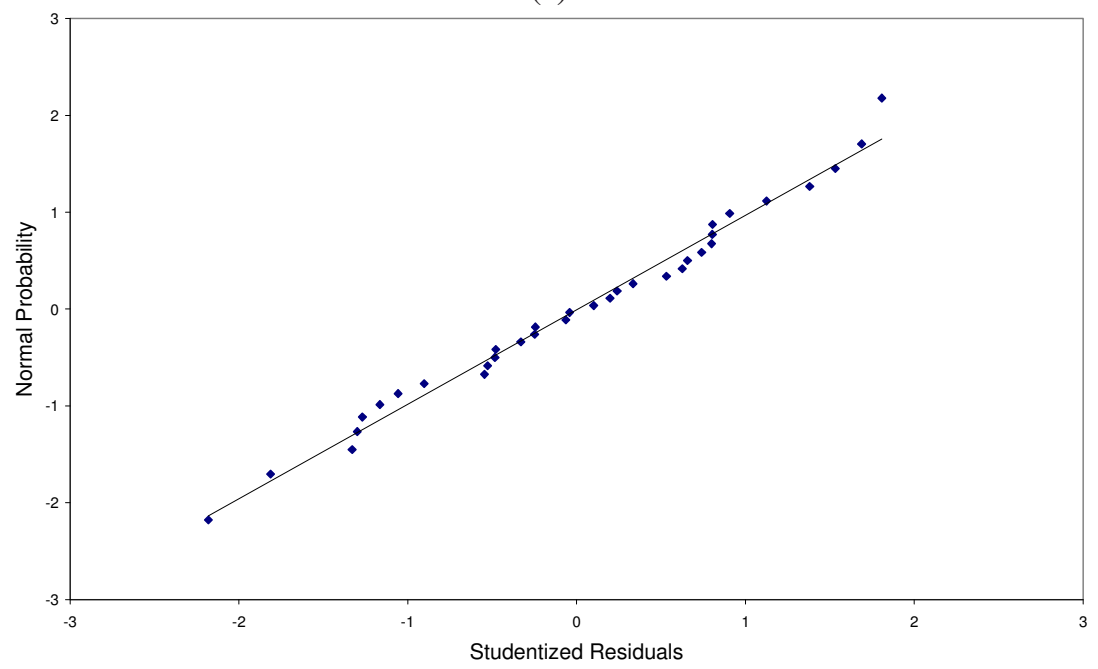

(b)

Figure 1 - Normal probability plot of the studentized residuals of Example 1. 


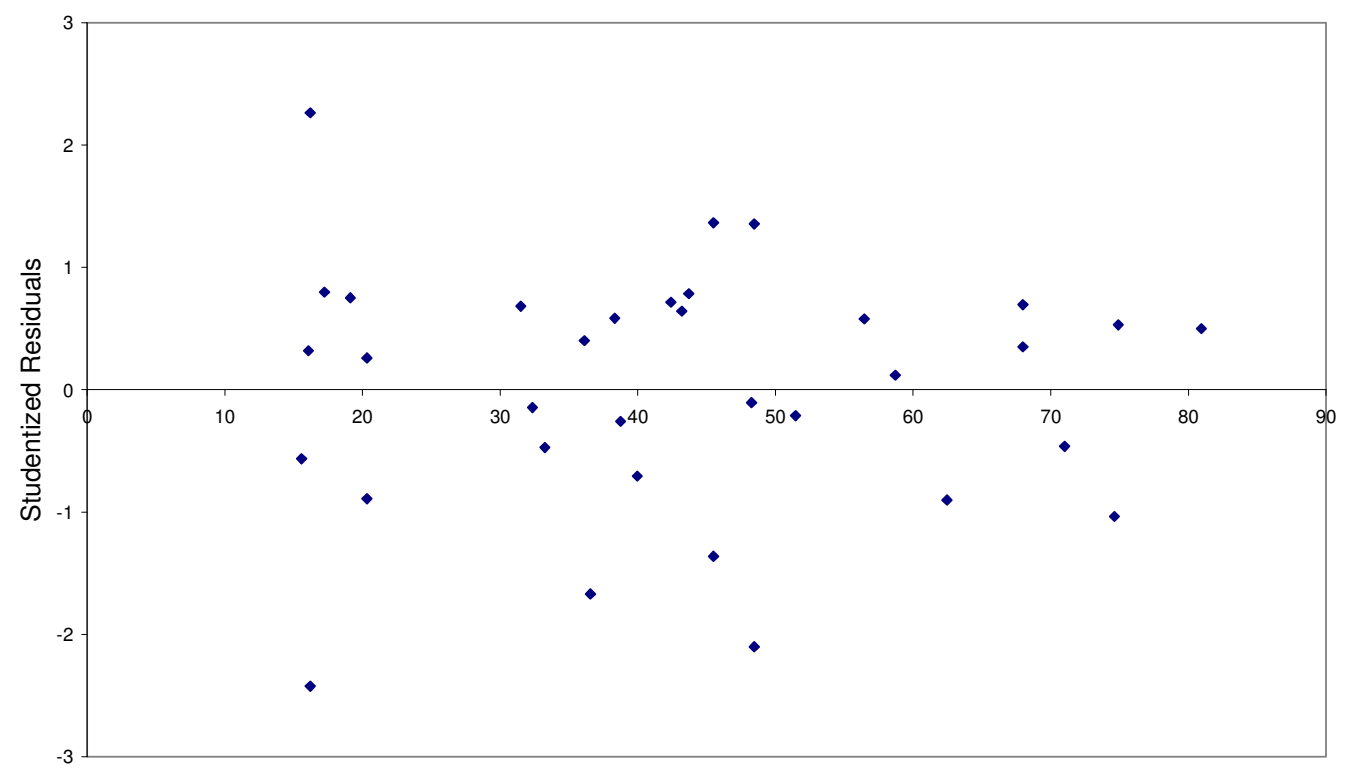

Predicted

(a)

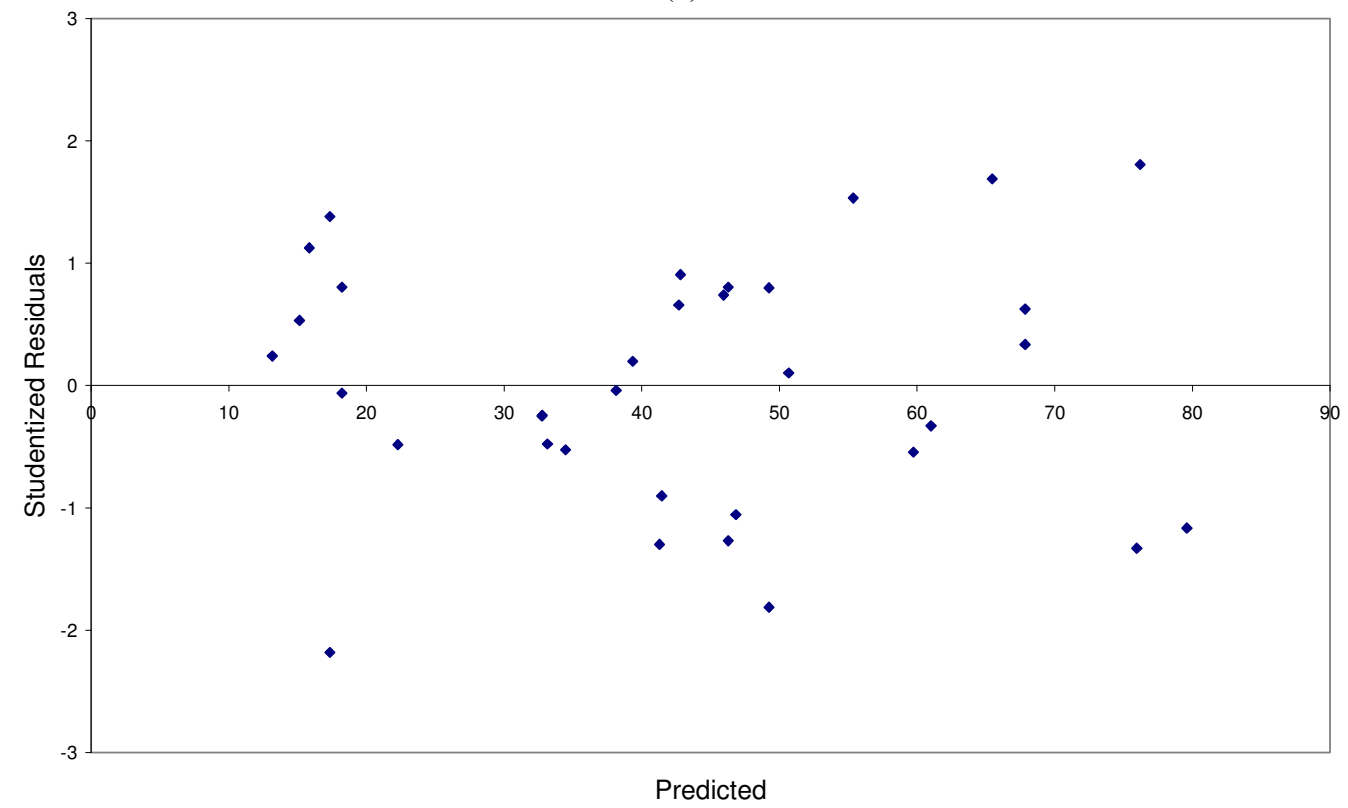

(b)

Figure 2 - Plot of studentized residuals versus fitted values of Example 1.

In the normal probability plots of the studentized residuals shown in Figure 1, we may observe that there isn't indication that the normality assumption should not be accepted, as there aren't points way off the alignment. 
In order to check the additivity of the models regarding the linear model, there are the plots of studentized residuals versus fitted values, shown in Figure 2.

The residuals shown in the plot from Figure 2 are randomly distributed around zero. Therefore, the adequacy of Final Model 1 (a) and the model obtained by Myers and Montgomery (b) were checked.

The fitted values shown in the plot from Figure 3 are randomly distributed around actual values. Therefore, the adequacy of Final Model 1 (a) and the model obtained by Myers \& Montgomery (b) were checked.

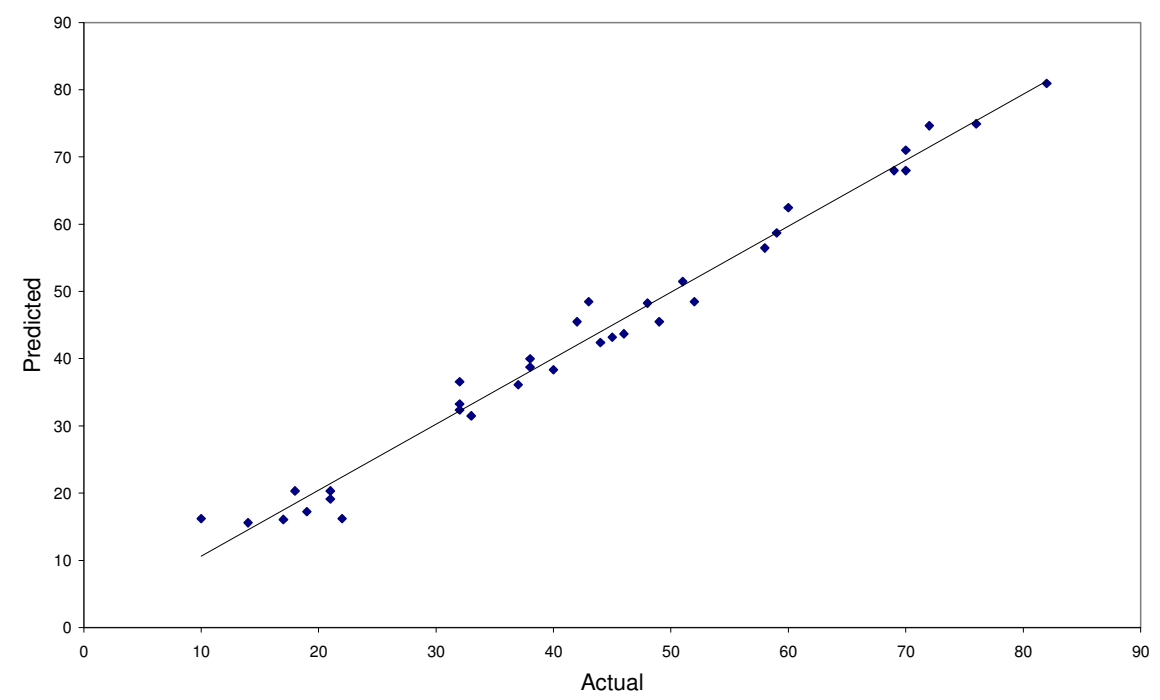

(a)

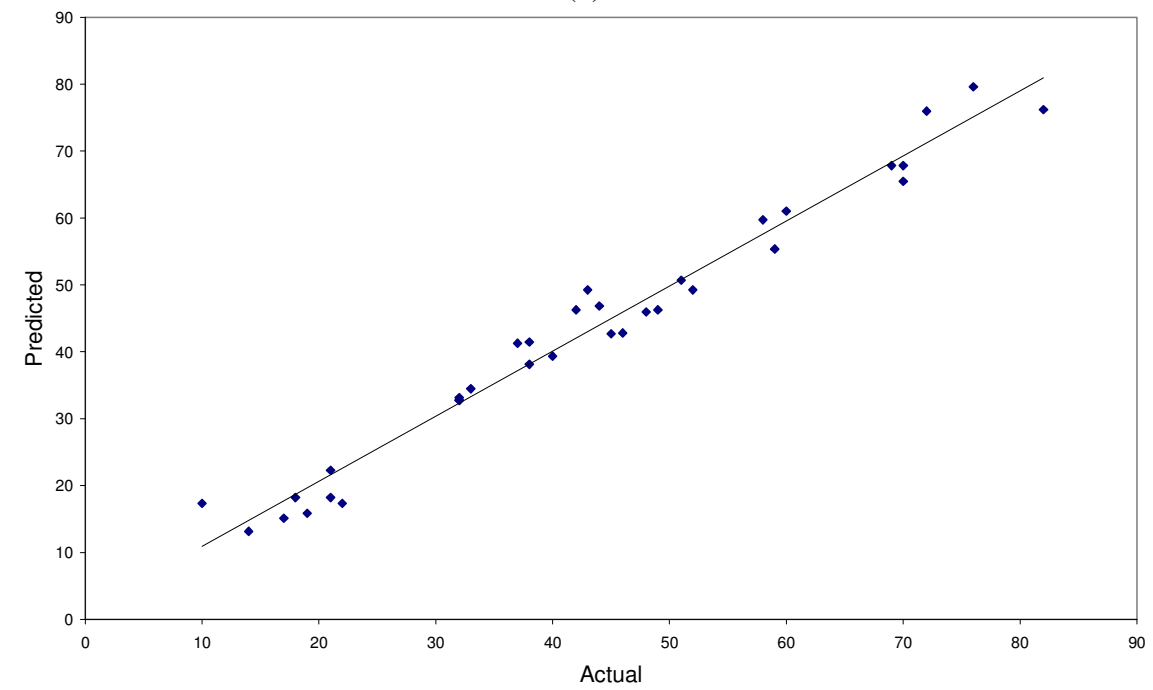

(b)

Figure 3 - Plot of fitted values versus actual values of Example 1. 


\subsection{Example 2}

The problem of Example 2 was presented by Cornell (2000) and Myers \& Montgomery (2002). Lauryl sulfate (A), cocamide (B), and lauramide (C) were ingredients in a shampoo whose proportionate values were varied in an experiment that was designed to study how shampoo foam height was functionally related to composition. The three ingredients made up 50\% of the shampoo, while the other constituents, which were held fixed in all blends, were water, perfume, and coloring agents.

Upper and lower bound constraints were placed on the ingredient or component proportions in the form $0.20 \leq A \leq 0.30,0.07 \leq B \leq 0.10$, and $0.13 \leq C \leq 0.20$, where $A+B+C=0.5$. The lower and upper bound constraints, when converted to the mixture components constraints in Eq. (1), are rescaled as $0.40 \leq x_{i} \leq 0.60,0.14 \leq x_{2} \leq 0.20$, and $0.26 \leq x_{3} \leq 0.40$. The experimenter's objective was to formulate a product with foam height in excess of $170 \mathrm{~mm}$. The authors use L-pseudocomponents and Table 6 presents the experiment.

Table 6 - The experiment of Example 2 with L-pseudocomponents for mixture components.

\begin{tabular}{|c|c|c|c|c|c|c|c|}
\hline Std & Run & $v_{1}$ & $v_{2}$ & $v_{3}$ & Height $(\mathrm{mm})$ & $\hat{y}_{1}$ & $\hat{y}_{2}$ \\
\hline 1 & 11 & 1.000 & 0.000 & 0.000 & 152.0 & 146.740 & 146.670 \\
2 & 12 & 1.000 & 0.000 & 0.000 & 140.0 & 146.740 & 146.670 \\
3 & 3 & 0.700 & 0.300 & 0.000 & 150.0 & 148.162 & 147.140 \\
4 & 6 & 0.700 & 0.300 & 0.000 & 145.0 & 148.162 & 147.140 \\
5 & 5 & 0.000 & 0.300 & 0.700 & 141.0 & 139.428 & 138.600 \\
6 & 2 & 0.000 & 0.300 & 0.700 & 138.0 & 139.428 & 138.600 \\
7 & 10 & 0.300 & 0.000 & 0.700 & 153.0 & 148.926 & 149.484 \\
8 & 4 & 0.300 & 0.000 & 0.700 & 147.0 & 148.926 & 149.484 \\
9 & 8 & 0.850 & 0.150 & 0.000 & 165.0 & 164.223 & 164.107 \\
10 & 7 & 0.650 & 0.000 & 0.350 & 170.0 & 170.276 & 169.052 \\
11 & 1 & 0.350 & 0.300 & 0.350 & 148.0 & 146.945 & 150.597 \\
12 & 13 & 0.750 & 0.075 & 0.175 & 175.0 & 171.203 & 173.145 \\
13 & 9 & 0.400 & 0.075 & 0.525 & 163.0 & 167.831 & 166.273 \\
\hline
\end{tabular}

The models selected by Cornell (2000) and Myers \& Montgomery (2002) both presented PRESS, MSE and $A I C_{c}$ equal to 657.08, 25.14 and 83.87, respectively, and the model by Cornell (2000) is shown in Eq. (20), while that by Myers \& Montgomery (2002) is shown in Eq. (21).

$$
\begin{aligned}
\hat{y}= & 94.90+235.05 v_{1}+411.42 v_{2}-839.41 v_{1} v_{2} \\
& -183.21 v_{1}^{2}-876.64 v_{2}^{2}+524.99 v_{1} v_{2}\left(v_{1}+v_{2}\right) \\
\hat{y}= & 146.74 v_{1}+370.32 v_{2}+94.90 v_{3}+745.43 v_{1} v_{2} \\
& +183.21 v_{1} v_{3}+876.64 v_{2} v_{3}-524.99 v_{1} v_{2} v_{3}
\end{aligned}
$$

Where

$\hat{y}_{1}$ : Response obtained by Cornell (2000) and Myers \& Montgomery (2002) models.

$\hat{y}_{2}$ : Response obtained by Final Model 2 in this article. 


\subsubsection{Model Selection}

All the candidate terms for the ME are the terms in Eq. (3). The model selected according to the $A I C_{c}$ criterion was the following:

$$
\begin{aligned}
\hat{y}= & 145.89 v_{1}+53.69 v_{2}+175.89 v_{3}+485.98 v_{1} v_{2} v_{3} \\
& +353.65 v_{1} v_{2}\left(v_{1}-v_{2}\right)+190.38 v_{1} v_{3}\left(v_{1}-v_{3}\right)
\end{aligned}
$$

Then, four models are considered non-different. The Model that presents the lowest PRESS (413.22) and MSE (17.09) was selected and named as the Base Model. The Base Model is shown in Eq. (22).

In this step of the methodology we will consider other models using the Base Model. For this, additional terms are generated from the terms of the Base Model. Table 7 presents the equivalent terms to the Base Model terms.

Table 7 - Equivalent Terms.

\begin{tabular}{|l|l|}
\hline Base Model Terms & Equivalent Terms \\
\hline & $\left(v_{2} v_{3}-v_{2}^{2} v_{3}-v_{2} v_{3}^{2}\right) ;$ \\
$\left(v_{1} v_{3}-v_{2}^{2} v_{3}-v_{1} v_{3}^{2}\right) ;$ \\
$\left(v_{1} v_{2}-v_{1}^{2} v_{2}-v_{1} v_{2}^{2}\right)$
\end{tabular}

Once all the candidate terms (Base Model terms and Equivalent Terms) for the ME model are known, we may then use the $A I C_{c}$ criterion again. The model selected is shown in Eq. (23). This model presents PRESS and MSE equal to 348.73 and 16.61, respectively.

$$
\hat{y}=146.67 v_{1}+301.14 v_{2}+134.47 v_{3}+180.23 v_{1}^{2} v_{3}-1698.94 v_{2}^{3}
$$

We will analyze models considered not different to the model of Eq. (23), as described at the start in Section 5.1.1. The Model that presents the lowest PRESS (348.73) and MSE (16.61) was selected and now, it should be Final Model 2.

Table 8 shows the $t$-Student test for the Final Model 2.

Comparing the models obtained by Cornell (2000) and Myers \& Montgomery (2002) and Final Model 2 obtained in this article, we observed that the application of the methodology led to a decrease of $46.93 \%$ in PRESS (from 657.08 to 348.73 ) and decrease of $33.93 \%$ in the MSE (from 25.14 to 16.61). It should be emphasized that Final Model 2 has five parameters and the models presented by Cornell (2000) and Myers \& Montgomery (2002) have seven parameters. 
Table 8 - Final Model 2 Test.

\begin{tabular}{|c|r|c|r|r|}
\hline Label & Estimate & Std. Error & $t$ value & $p$-value \\
\hline$v_{1}$ & 146.67 & 2.61705 & 56.046 & $<0.0001$ \\
$v_{2}$ & 301.14 & 32.6991 & 9.209 & $<0.0001$ \\
$v_{3}$ & 134.47 & 4.15726 & 32.346 & $<0.0001$ \\
$v_{1}^{2} v_{3}$ & 180.23 & 30.1357 & 5.981 & 0.0003 \\
$v_{2}^{3}$ & -1698.94 & 359.383 & -4.727 & 0.0015 \\
\hline
\end{tabular}

\subsubsection{Response Optimization}

In the Example 2, a response of exceeding $170 \mathrm{~mm}$ is desirable. Several formulations may result in future a response prediction greater than $170 \mathrm{~mm}$. Consequently, a desirable objective is to maximize the expected value for a future response.

The problem may then be formulated as follows:

$$
\max E[\hat{y}(\boldsymbol{w})]=\boldsymbol{w}^{\prime} \hat{\boldsymbol{\beta}}
$$

subject to:

$$
\begin{aligned}
& v_{1}+v_{2}+v_{3}=1 \\
& 0 \leq v_{1} \leq 1 \\
& 0 \leq v_{2} \leq 0.3 \\
& 0 \leq v_{3} \leq 0.7
\end{aligned}
$$

Table 9 presents the optimal values for the components proportions, in L-pseudocomponents $\left(v_{i}\right)$ and in actual values $(A, B$ and $C)$. Table 10 compares the PRESS, MSE, $A I C_{c}$, the response prediction and the variance of a new response for three models. Analyzing the Table 9, we observe that the model obtained in this article presents lower value for PRESS, MSE and $A I C_{c}$, emphasizing that was obtained a higher response prediction with a lower variance of a new response.

Table 9 - Solution for the maximization problem of Example 2.

\begin{tabular}{|c|c|c|c|}
\hline Model & $\begin{array}{c}v_{1} \\
(A)\end{array}$ & $\begin{array}{c}v_{2} \\
(B)\end{array}$ & $\begin{array}{c}v_{3} \\
(C)\end{array}$ \\
\hline Cornell, & 0.6040 & 0.0855 & 0.3105 \\
Myers \& Montgomery & $(0.2604)$ & $(0.0786)$ & $(0.1611)$ \\
\hline Final Model 2 & 0.6103 & 0.1397 & 0.2500 \\
& $(0.2610)$ & $(0.0840)$ & $(0.1550)$ \\
\hline
\end{tabular}

\subsubsection{Model Adequacy}

Figures 4 and 5 present the diagnosis plots used to check the adequacy of the Final Model 2 (a) and the models obtained by Cornell and, Myers \& Montgomery (b). 
Table 10 - Comparison of three models.

\begin{tabular}{|c|c|c|c|c|c|}
\hline Model & $P R E S S$ & $M S E$ & $A I C_{c}$ & $\operatorname{var}[\hat{y}(w)]$ & $E[\hat{y}(w)]$ \\
\hline $\begin{array}{c}\text { Cornell, } \\
\text { Myers \& Montgomery }\end{array}$ & 657.08 & 25.14 & 83.87 & 37.7445 & 174.1457 \\
\hline Final Model 2 & 348.73 & 16.61 & 56.22 & 27.8203 & 177.3530 \\
\hline
\end{tabular}

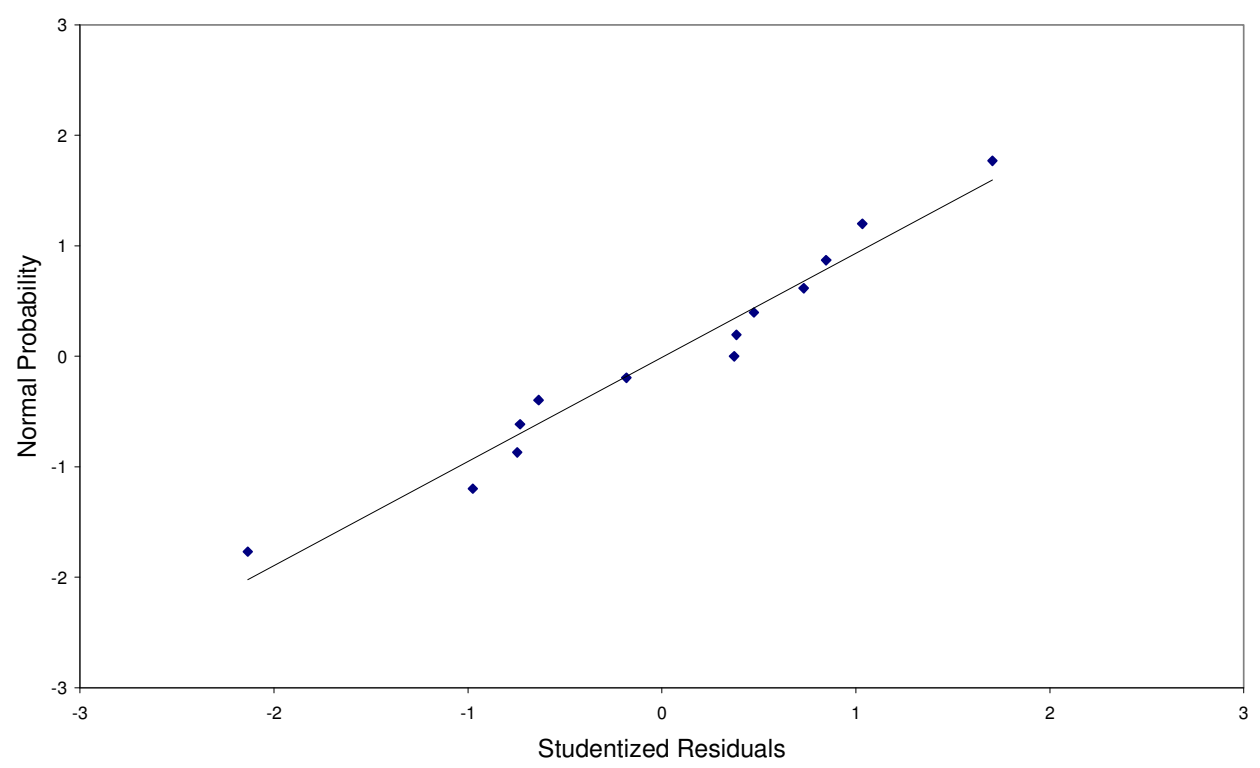

(a)

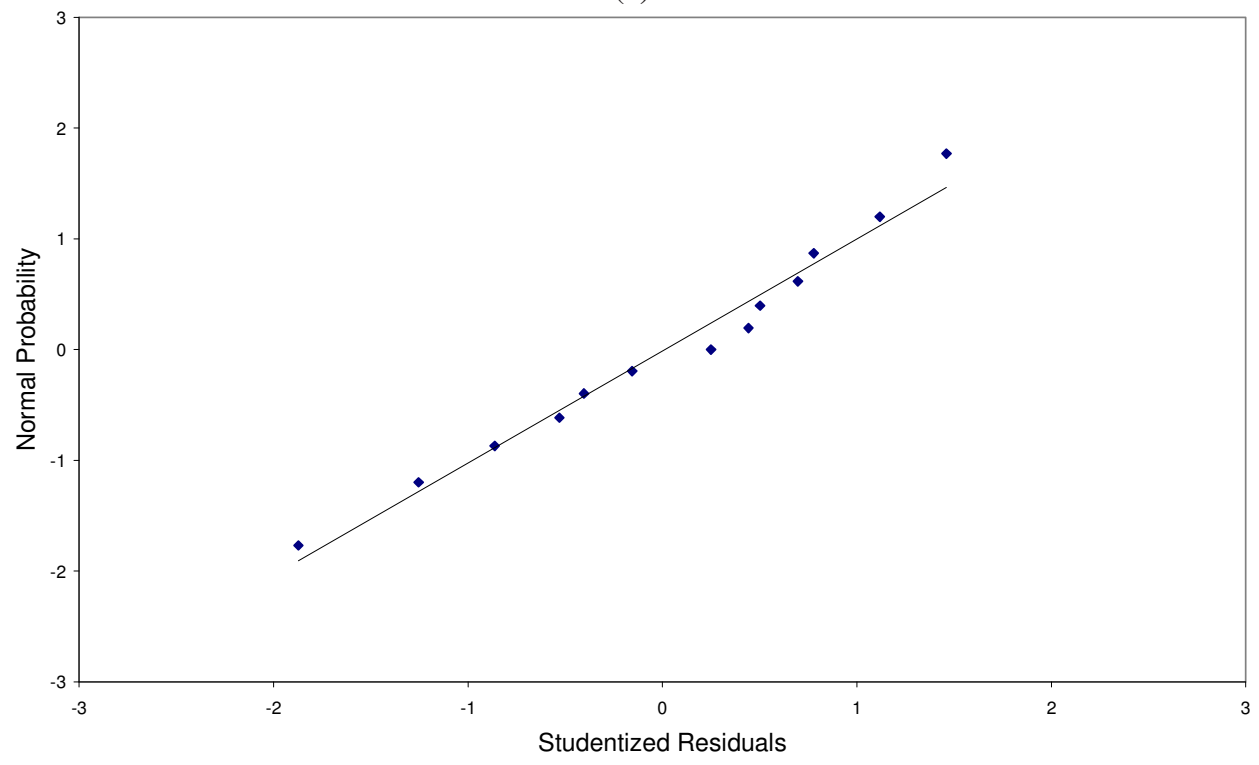

(b)

Figure 4 - Normal probability plot of the studentized residuals of Example 2. 
In the normal probability plot of the studentized residuals shown in Figure 4, we may observe that there isn't indication that the normality assumption should not be accepted, as there aren't points way off the alignment.

In order to check the additivity of the model regarding the linear model, there is the plot of studentized residuals versus fitted values, shown in Figure 5.

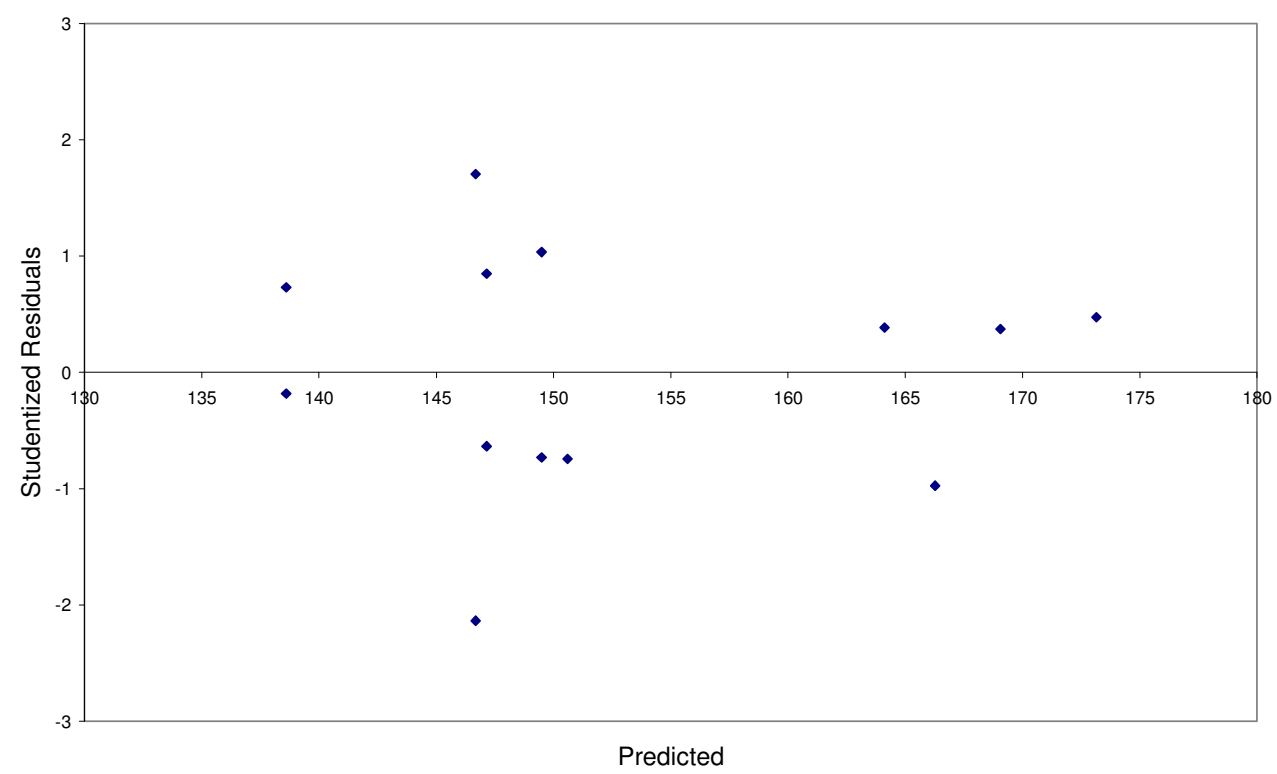

(a)

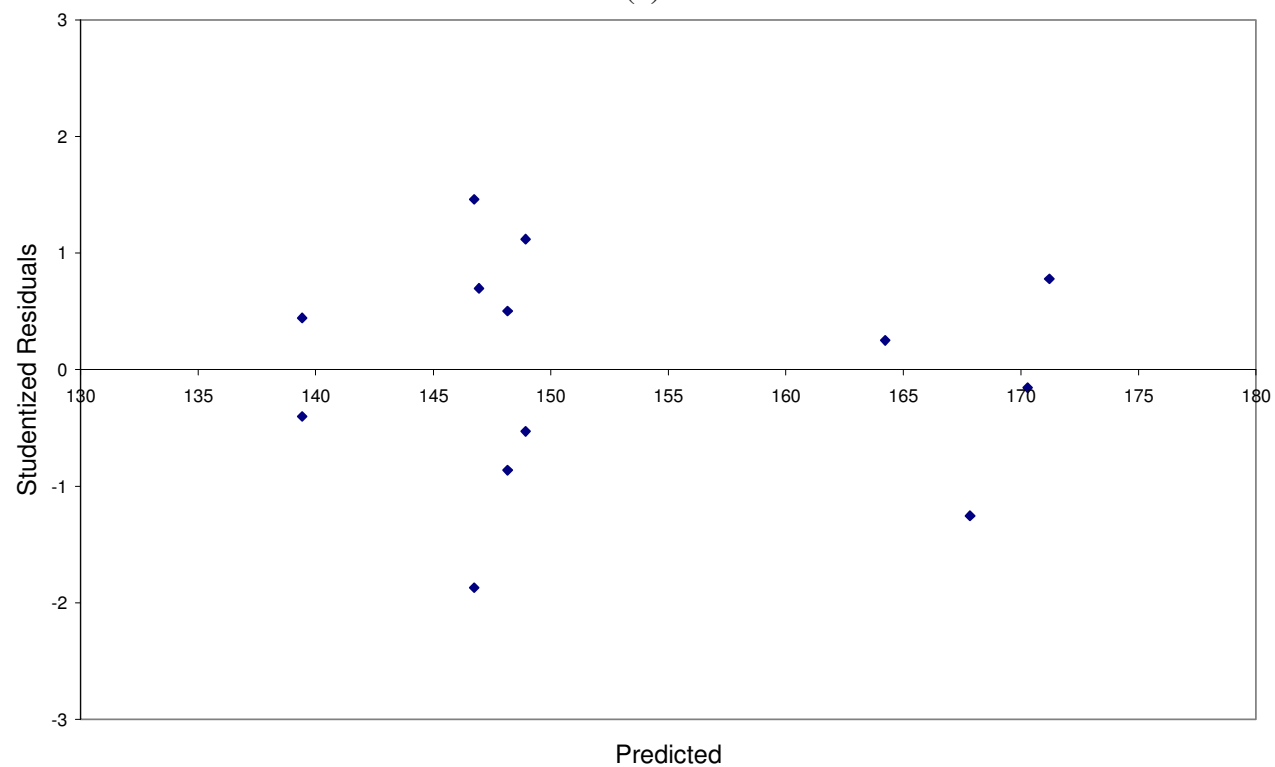

(b)

Figure 5 - Plot of studentized residuals versus fitted values of Example 2. 
The residuals shown in the plot from Figure 5 are randomly distributed around zero. Therefore, the adequacy of Final Model 2 (a) and the models obtained by Cornell and, Myers \& Montgomery (b) were checked.

The fitted values shown in the plot from Figure 6 are randomly distributed around actual values. Therefore, the adequacy of Final Model 2 (a) and the model obtained by Cornell and, Myers \& Montgomery (b) were checked.

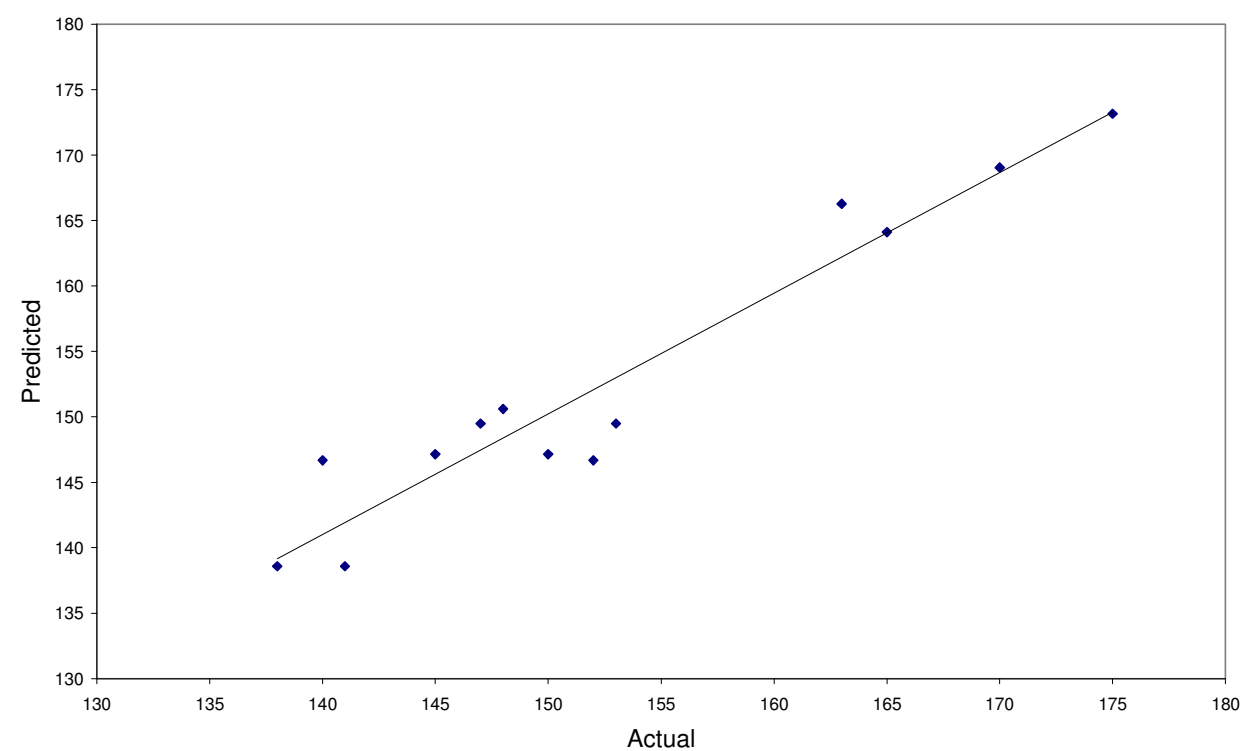

(a)

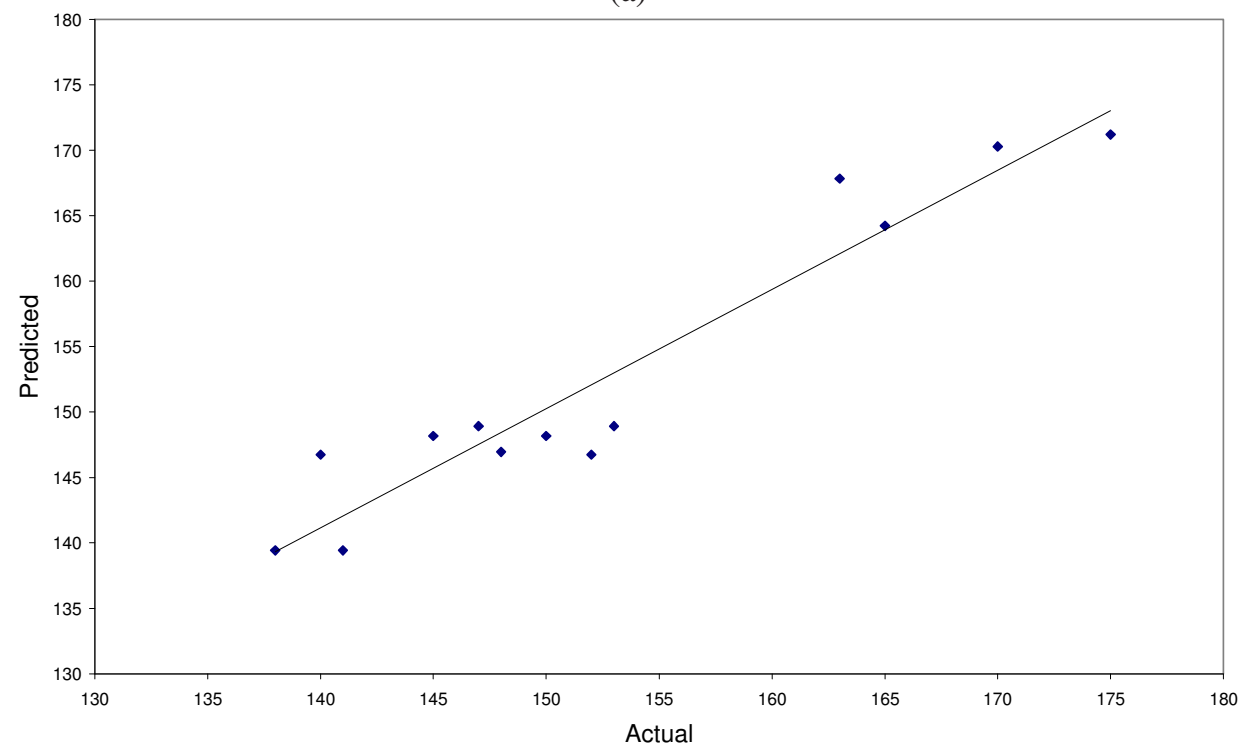

(b)

Figure 6 - Plot of fitted values versus actual values of Example 2. 
Below we present the steps of the methodology proposed in this article:

Step 1: Choose a full model based on Scheffé's canonical polynomials for cases of ME, shown in Eq. (3), or choose a full combined model for cases of MPE, shown in Eq. (5), to obtain all the candidate terms.

Step 2: Use the $A I C_{c}$ criterion and select the model that provides the lowest $A I C_{c}$, according to the number of parameters.

Step 3: Calculate the $A I C_{c}$ differences between the candidate models and the model that provides the lowest $A I C_{c}$ and select the non-different models.

Step 4: Analyze the non-different models, and choose the model that provides the lowest MSE and PRESS. This model is now named as the Base Model.

Step 5: Determine all terms that are equivalent to the Base Model terms, and create all the candidate terms.

Step 6: Use the $A I C_{c}$ criterion again, and select the model that provides the lowest $A I C_{c}$ according to the number of parameters.

Step 7: Calculate the $A I C_{c}$ differences between the candidate models and the model that provides the lowest $A I C_{c}$ and select the non-different models again.

Step 8: Analyze the non-different models, and choose the Final Model that provides the lowest MSE and PRESS.

\section{SIMULATION STUDY}

A small simulation study is now presented once we know the true model leading to the responses. Considering the experiment of the Table 11 and the true model shown in Eq. (24), we developed a routine in Matlab ${ }^{\circledR}$ to generate the normal responses for this experiment and to select the model using the proposed methodology.

$$
y=150 v_{1}+300 v_{2}+150 v_{3}+50 v_{1} v_{2}-50 v_{1} v_{3}+250 v_{1}^{2} v_{3}-650 v_{2}^{2}+\varepsilon
$$

where $\varepsilon$ is the random errors with normal distribution, i.e. $\varepsilon \sim N\left(0, \sigma^{2}\right)$.

In the simulation study we generated 1,000 experiments with the model shown in Eq. (24), considering $\sigma$ equal to 0.5 up to 1.5 . Using the proposed methodology, the Table 12 shows the identified models with the same terms of the model shown in Eq. (24).

\section{CONCLUSIONS}

In this article, the statistical techniques necessary for the planning and analysis of mixture experiments with or without process variables were gathered and a methodology for selecting models in MPE and ME was presented with two examples.

The use of Information Theory constituted an evolution in ME and MPE. Multicollinearity may cause the estimators of model coefficients to be instable and very inflated. Therefore, certain 
Table 11 - The experiment of the Simulation Study.

\begin{tabular}{|c|c|c|c|}
\hline Std & $v_{1}$ & $v_{2}$ & $v_{3}$ \\
\hline 1 & 1.000 & 0.000 & 0.000 \\
2 & 1.000 & 0.000 & 0.000 \\
3 & 0.700 & 0.300 & 0.000 \\
4 & 0.700 & 0.300 & 0.000 \\
5 & 0.000 & 0.300 & 0.700 \\
6 & 0.000 & 0.300 & 0.700 \\
7 & 0.300 & 0.000 & 0.700 \\
8 & 0.300 & 0.000 & 0.700 \\
9 & 0.850 & 0.150 & 0.000 \\
10 & 0.650 & 0.000 & 0.350 \\
11 & 0.350 & 0.300 & 0.350 \\
12 & 0.750 & 0.075 & 0.175 \\
13 & 0.400 & 0.075 & 0.525 \\
\hline
\end{tabular}

Table 12 - $\sigma$ versus Identified Models.

\begin{tabular}{|c|c|}
\hline$\sigma$ & Identified Models \\
\hline 0.5 & 1,000 \\
0.6 & 1,000 \\
0.7 & 1,000 \\
0.8 & 1,000 \\
0.9 & 998 \\
1.0 & 994 \\
1.1 & 980 \\
1.2 & 962 \\
1.3 & 952 \\
1.4 & 932 \\
1.5 & 926 \\
\hline
\end{tabular}

terms of the model may be significant in the presence of some terms and not significant in the presence of other terms. In this context, stepwise forward and backward selection may result in arbitrary selection of variables that belong to the model (Harrell, 2001). An alternative technique was to consider all possible combinations of terms in the full model and the number of parameters and to use selection criteria for models based on Information Theory. From the results obtained in this article, we concluded that the use of the AICc information criterion may result in lower PRESS and MSE.

Finally, a model selection methodology in ME and MPE was presented. In the first stage of the methodology, a Base Model was fitted using the AICc criterion. In the following stage, a better model was obtained, taking into account, besides the Base Model terms, all the Equivalent Terms of the Base Model, also using the AICc criterion for the selection of the proposed model terms. 
We may then conclude that the second stage of the methodology provided models that were better than the Base Model and also better than the models obtained previously.

\section{REFERENCES}

[1] AKaike H. 1973. Information theory and an extension of the maximum likelihood principle, in Mehra RK \& Csaki F. (editors), Second International Symposium on Information Theory. Akademiai Kiado, Budapest.

[2] Anderson-Cook CM, Goldfarb HB, Borror CM, Montgomery DC, Canter KG \& TWIST JN. 2004. Mixture and mixture-process variable experiments for pharmaceutical applications. Pharmaceutical Statistics, 3(4): 247-260.

[3] Borror CM, Montgomery DC \& Myers RH. 2002. Evaluation of statistical designs for experiments involving noise variables. Journal of Quality Technology, 34(1): 54-70.

[4] Burnham KP \& Anderson DR. 2002. Model Selection and Multimodel Inference: A Practical Information-Theoretical Approach. Second edition, Springer, New York.

[5] Chung PJ, Goldfarb HB \& Montgomery DC. 2007. Optimal Designs for Mixture-Process Experiments with Control and Noise Variables. Journal of Quality Technology, 39: 179-190.

[6] Chung PJ, Goldfarb HB, Montgomery DC \& Borror CM. 2009. Optimal Designs for Mixture-Process Experiments Involving Continuous and Categorical Noise Variables. Quality Technology \& Quantitative Management, 6(4): 451-470.

[7] CORnell JA. 1995. Fitting models to data from mixture experiments containing other factors. Journal of Quality Technology, 27(1): 13-33.

[8] Cornell JA. 2000. Fitting a slack-variable model to mixture data: some questions raised. Journal of Quality Technology, 32(2): 133-147.

[9] Cornell JA. 2002. Experiments with Mixtures: Designs, Models and the Analysis of Mixture Data. Third edition, John Wiley and Sons, New York.

[10] Cornell JA \& Gorman JW. 2003. Two New Mixture Models: Living With Collinearity but Removing its Influence. Journal of Quality Technology, 35: 78-88.

[11] Dal Bello LHA. 2010. Modelagem em Experimentos Mistura-Processo para Otimização de Processos Industriais. Doctoral Thesis, PUC, Rio de Janeiro.

[12] Dal Bello LHA \& Vieira AFC. 2011a. Optimization of a product performance using mixture experiments. Journal of Applied Statistics, 38(8): 1701-1715.

[13] Dal Bello LHA \& VIeIRA AFC. 2011b. Tutorial for mixture-process experiments with an industrial application. Pesquisa Operacional, 31(3): 1-21.

[14] Goldfarb HB, Borror CM \& Montgomery DC. 2003. Mixture-Process Variable Experiments with Noise Variables. Journal of Quality Technology, 35: 393-405.

[15] Goldfarb HB, Borror CM, Montgomery DC \& Anderson-Cook CM. 2004a. ThreeDimensional Variance Dispersion Graphics for Mixture-Process Experiments. Journal of Quality Technology, 36: 109-124.

[16] Goldfarb HB, Borror CM, Montgomery DC \& Anderson-Cook CM. 2004b. Evaluating Mixture-Process with Control and Noise Variables. Journal of Quality Technology, 36: 245-262. 
[17] Goos P \& Donev AN. 2006. The D-Optimal Design of Blocked Experiments with Mixture Components. Journal of Quality Technology, 38: 319-332.

[18] Goos P \& Donev AN. 2007. Tailor-Made Split-Plot Designs for Mixture and Process Variables. Journal of Quality Technology, 39: 326-339.

[19] HARRELl FE JR. 2001. Regression Modeling Strategies with Applications to Linear Models, Logistic Regression, and Survival Analysis. Springer-Verlag, New York.

[20] Hurvich CM \& TSAI C-L 1989. Regression and time series model selection in small samples. Biometrika, 76: 297-307.

[21] KhuRi AI. 2005. Slack Variable Models Versus Scheffés Mixture Models. Journal of Applied Statistics, 32: 887-908.

[22] Kowalski SM, Cornell JA \& Vining GG. 2002. Split-Plot Designs and Estimation Methods for Mixture Experiments with Process Variables. Technometrics, 44: 72-79.

[23] Myers RH \& Montgomery DC. 2002. Response Surface Methodology: Process and Product Optimization Using Designed Experiments. Second edition. John Wiley and Sons, New York.

[24] PIEPEL GF. 2004. 50 Years of mixture experiment research: 1955-2004, in KHURI AI. (Editor). Response Surface Methodology and Related Topics. World Scientific Publishing, Singapore, 283327.

[25] Piepel GF. 2007. A Component Slope Linear Model for Mixture Experiments. Quality Technology \& Quantitative Management, 4(3): 331-343.

[26] PrescotT P. 2004. Modeling in Mixture Experiments Including Interactions with Process Variables. Quality Technology \& Quantitative Management, 1(1): 87-103.

[27] SAhni NS, PiePel GF \& NÆS T. 2009. Product and Process Improvement Using Mixture-Process Variable Methods and Optimization Techniques. Journal of Quality Technology, 41(2): 181-197.

[28] SCHEFFÉ H. 1958. Experiments with mixture. Journal of the Royal Statistical Society, B20: 344-366. 